\title{
THE SEMIGROUP OF A QUASI-ORDINARY HYPERSURFACE
}

\author{
PEDRO DANIEL GONZÁLEZ PÉREZ \\ Université de Paris 7, Institut de Mathématiques, Equipe Géométrie et Dynamique, \\ Case 7012, 2, Place Jussieu, 75005 Paris, France (gonzalez@math.jussieu.fr)
}

\begin{abstract}
An analytically irreducible hypersurface germ $(S, 0) \subset\left(\boldsymbol{C}^{d+1}, 0\right)$ is quasi-ordinary if it can be defined by the vanishing of the minimal polynomial $f \in \boldsymbol{C}\{X\}[Y]$ of a fractional power series in the variables $X=\left(X_{1}, \ldots, X_{d}\right)$ which has characteristic monomials, generalizing the classical NewtonPuiseux characteristic exponents of the plane-branch case $(d=1)$. We prove that the set of vertices of Newton polyhedra of resultants of $f$ and $h$ with respect to the indeterminate $Y$, for those polynomials $h$ which are not divisible by $f$, is a semigroup of rank $d$, generalizing the classical semigroup appearing in the plane-branch case. We show that some of the approximate roots of the polynomial $f$ are irreducible quasiordinary polynomials and that, together with the coordinates $X_{1}, \ldots, X_{d}$, provide a set of generators of the semigroup from which we can recover the characteristic monomials and vice versa. Finally, we prove that the semigroups corresponding to any two parametrizations of $(S, 0)$ are isomorphic and that this semigroup is a complete invariant of the embedded topological type of the germ $(S, 0)$ as characterized by the work of Gau and Lipman.
\end{abstract}

Keywords: quasi-ordinary singularities; topological type; semigroup; discriminant

AMS 2000 Mathematics subject classification: Primary 14M25; 32S25

\section{Introduction}

The set of intersection multiplicities at the origin of a plane branch $(\mathcal{C}, 0)$ with those curves that do not contain this branch as a component is a sub-semigroup of $\boldsymbol{Z}_{\geqslant 0}$, which is, in fact, a complete invariant of the embedded topological type of the germ $(\mathcal{C}, 0) \subset\left(\boldsymbol{C}^{2}, 0\right)$ (see $[\mathbf{R e}]$ ). If $f \in \boldsymbol{C}\{X\}[Y]$ is a Weierstrass polynomial defining the germ $\mathcal{C}$, we can build a system of generators of the semigroup by considering the curves defined by $X=0$ and by the vanishing of some of the approximate roots of the polynomial $f$. If the curve $X=0$ is not contained in the tangent cone of the germ $(\mathcal{C}, 0)$, this set of generators is the minimal one (see $[\mathbf{Z 2}, \mathbf{A}-\mathbf{M} 1, \mathbf{A}-\mathbf{M} 2, \mathbf{P P} 1, \mathbf{G}-\mathbf{P}]$ ).

In this paper we generalize some of the above results to the more general class of analytically irreducible quasi-ordinary hypersurface germs. A germ of complex analytic variety $(S, o)$ is quasi-ordinary if there exists a finite morphism $(S, o) \rightarrow\left(\boldsymbol{C}^{d}, 0\right)$ such that the discriminant locus is contained (germ wise) in a normal crossing divisor. Quasiordinary hypersurface singularities arise classically in Jung's approach of analysing a surface singularity by using embedded resolution of the discriminant of any of its finite projections to a smooth surface (see $[\mathbf{L 2}, \mathbf{W}])$. 
A quasi-ordinary hypersurface germ can be defined by an equation $f=0$, where $f \in C\{X\}[Y]$ is a quasi-ordinary polynomial, i.e. a monic polynomial such that the discriminant $\Delta_{Y} f$ is of the form $X^{\delta} \varepsilon$ for a unit $\varepsilon$ (where $X$ denotes $\left(X_{1}, \ldots, X_{d}\right)$ ). The Jung-Abhyankar theorem implies that the roots of quasi-ordinary polynomials, called quasi-ordinary branches, are fractional power series in the ring $C\left\{X^{1 / n}\right\}$ for some positive integer $n$ (see $[\mathbf{J}, \mathbf{A} \mathbf{1}]$ ). Since the difference $\zeta-\zeta^{\prime}$ of any two roots of $f$ divides the discriminant, it must be of the form $X^{\lambda} \epsilon$, where $\epsilon \in C\left\{X^{1 / n}\right\}$ is a unit and $\lambda$ is a $d$ tuple of non-negative rationals. The monomials $X^{\lambda}$ so obtained are called characteristic monomials.

Lipman builds an inversion lemma that associates to any quasi-ordinary branch $\zeta$ a normalized quasi-ordinary branch parametrizing the same germ whose characteristic monomials are obtained from those of $\zeta$ by an inversion formulae similar to that of the plane-curve case (see $[\mathbf{L} \mathbf{1}]$ and the appendix of $[\mathbf{G a u}]$ ). Being normalized is a technical condition that in the plane-curve case means that the projection $(X, Y) \mapsto X$ is transversal. In the two-dimensional case, Lipman proved that the characteristic monomials of a normalized quasi-ordinary branch are an analytical invariant of the surface (see $[\mathbf{L} 1, \mathbf{L} 3]$ ). Luengo gives another proof of this result (see $[\mathbf{L u}]$ ). Lipman remarked, using general results of Zariski on saturation of local rings, that the characteristic monomials of a normalized quasi-ordinary branch determine the topological type of the germ it parametrizes (see $[\mathbf{L} 4]$ and also $[\mathbf{O h}]$ for another proof); Gau proved the converse: these monomials define a complete invariant of the embedded topological type of the germ. Gau's proof involves some results of Lipman on topological invariants of quasi-ordinary singularities: the description of the local divisor class group in terms of the characteristic monomials (see [Gau, L4]).

When $f$ is an irreducible quasi-ordinary polynomial, we can generalize some of the properties of the intersection multiplicity of plane-curve germs by studying the combinatorial structure of the set of compact faces of the Newton polyhedra of the resultants with respect to the indeterminate $Y$ of the polynomial $f$ and polynomials in the ring $C\{X\}[Y]$. In particular, we prove that the set of vertices of Newton polyhedra of the resultant $\operatorname{Res}_{Y}(f, h)$, for those polynomials $h$ which are not divisible by $f$, is a semigroup of rank $d$ (see Theorem 3.6 and Corollary 3.7). When $d=1$, we obtain the classical semigroup associated a plane branch. In general, we prove that the Newton polyhedra of the resultants of $f$ and of the minimal polynomials of some suitable truncations of the series $\zeta$ together with the coordinates $X_{1}, \ldots, X_{d}$, have only one vertex and that these vertices generate the semigroup. The same result holds when we replace the minimal polynomials of the truncations of $\zeta$ by approximate roots of $f$ of the same degrees. We prove that these approximate roots are irreducible quasi-ordinary polynomials and that their roots are fractional power series that coincide up to prescribed terms, which are some of the characteristic monomials, with the series $\zeta$ (see Proposition 4.3). The set of generators of the semigroup is related to the characteristic monomials by formulae similar to those that appear in the plane-branch case. Relevant to these formulae and to the properties of the semigroup are a set of integers and rank- $d$ lattices determined by the characteristic 
monomials, which we use to give properties characterizing those semigroups associated to a quasi-ordinary branch.

In the last section we use Theorem 3.6 and Corollary 3.7 to prove that the semigroups associated to a quasi-ordinary branch and its normalized quasi-ordinary branch by the inversion lemma of Lipman are isomorphic. As a consequence, we give another proof of the inversion formulae relating their characteristic monomials. Then we prove Theorem 5.3, which states that the semigroup we build from a quasi-ordinary branch is a complete invariant of the embedded topological type (as characterized by Gau): two quasi-ordinary branches parametrizing the same hypersurface germ have isomorphic semigroups and this semigroup determines the normalized characteristic exponents. Up to Theorem 5.3, which uses the characterization of the topological type, the results of this work are valid also in the algebroid case when we replace $\boldsymbol{C}\{X\}$, the ring of germs of holomorphic functions at the origin of the affine complex space, by the ring of formal power series with coefficients in an algebraically closed field of characteristic zero.

The semigroup of a quasi-ordinary hypersurface germ determines an embedded resolution of the germ, re-embeded in an affine complex space of larger dimension, by a method generalizing that of Goldin and Teissier in the plane-branch case (see [G-T, GP3]).

\section{Quasi-ordinary hypersurface singularities}

A germ of complex analytic variety $(S, o)$ is a quasi-ordinary singularity if there exists a finite morphism $(S, o) \rightarrow\left(\boldsymbol{C}^{d}, 0\right)$ (called a quasi-ordinary projection) such that the discriminant locus is contained (germ wise) in a normal crossing divisor. This means that there exist some analytical coordinates $X=\left(X_{1}, \ldots, X_{d}\right)$ at the origin such that the projection is unramified over the torus $X_{1} \cdots X_{d}=0$ in a neighbourhood of the origin. The finite map $(S, o) \rightarrow\left(\boldsymbol{C}^{d}, 0\right)$ corresponds algebraically to a local homomorphism $\boldsymbol{C}\{X\} \rightarrow R$ of their analytic algebras, which gives $R$ the structure of finite $\boldsymbol{C}\{X\}$-module. The germ $(S, o)$ is a quasi-ordinary hypersurface if $R$ is generated by one element. In this case, we have a surjection $C\{X\}[Y] \rightarrow R$ corresponding geometrically to an embedding $(S, o) \hookrightarrow\left(\boldsymbol{C}^{d} \times \boldsymbol{C}, 0\right)$. The kernel of the homomorphism above is a principal ideal generated by a monic polynomial $f \in C\{X\}[Y]$ such that $f(0, Y)=Y^{n}$ (where $n=\operatorname{deg} f$ is also equal to the degree of the quasi-ordinary projection) and the discriminant $\Delta_{Y} f$ of $f$ with respect to the variable $Y$ is of the form $X^{\delta} H$ for a unit $H$ in $C\{X\}$. We say that a polynomial $f$ in $\boldsymbol{C}\{X\}[Y]$ satisfying these conditions is quasi-ordinary, since it defines a quasi-ordinary hypersurface for the projection $(X, Y) \mapsto X$. The Jung-Abhyankar theorem (see $[\mathbf{J}]$ or $[\mathbf{A} 1$, Theorem 3] for an algebraic proof) implies that the roots of the quasi-ordinary polynomial $f$ are fractional power series in the $\operatorname{ring} C\left\{X^{1 / k}\right\}$ for some positive integer $k$, where $X^{1 / k}=\left(X_{1}^{1 / k}, \ldots, X_{d}^{1 / k}\right)$. The roots of quasi-ordinary polynomials are called quasi-ordinary branches. Any difference of two distinct roots of $f$ divides the discriminant in $C\left\{X^{1 / k}\right\}$, thus it is necessarily of the form $\zeta^{(s)}-\zeta^{(t)}=X^{\lambda_{s t}} H_{s t}$, where $H_{s t}$ is a unit in $C\left\{X^{1 / k}\right\}$. When $d=1$, the exponents $\lambda_{s t}$ obtained are the classical Newton-Puiseux characteristic exponents of the (analytically) irreducible components of the germ $(S, o)$ in general coordinates and the orders of coincidence of the fractional parametrizations of distinct components. 
If the polynomial $f$ is irreducible, we can take $k=n:=\operatorname{deg} f$ (see [L4, p. 52] and [GP1, Remarque 1]). The analytic algebra $R=C\{X\}[Y] /(f)$ can be viewed as the subring $\boldsymbol{C}\{X\}[\zeta]$ of $\boldsymbol{C}\left\{X^{1 / n}\right\}$ for $\zeta$ any root of $f$. If $L$ (respectively, $L_{n}$ ) is the field of fractions of $\boldsymbol{C}\{X\}$ (respectively, of $\boldsymbol{C}\left\{X^{1 / n}\right\}$ ), the field extension $L \subset L_{n}$ is finite and Galois. Its Galois group is obtained from the action of $d$-tuples $\left(\eta_{1}, \ldots, \eta_{d}\right)$ of $n$th roots of unity given by $X_{i}^{1 / n} \mapsto \eta_{i} X_{i}^{1 / n}$ for $i=1, \ldots, d$. It follows that the field extension obtained from $R \subset C\left\{X^{1 / n}\right\}$ by taking fields of fractions is Galois, and therefore the roots of $f$ are the conjugates of $\zeta$ by the above action. The fractional monomials $X^{\lambda_{s t}}$ (respectively, the vector exponents $\lambda_{s t}$ ) are called characteristic or distinguished monomials (respectively, exponents) of the quasi-ordinary branch $\zeta$.

If $f$ is a reduced quasi-ordinary polynomial, it follows from the geometrical definition that all its irreducible factors are quasi-ordinary polynomials.

Definition 2.1 (cf. [GP2]). The polynomials $f^{(i)}$ and $f^{(j)}$ in $\boldsymbol{C}\{X\}[Y]$ have order of coincidence $\lambda_{(i, j)}$ if $f^{(i)} f^{(j)}$ is a quasi-ordinary polynomial and $\lambda_{(i, j)}$ is the largest exponent of the set $\left\{\lambda_{s t} / f^{(i)}\left(\zeta^{(s)}\right)=0, f^{(j)}\left(\zeta^{(t)}\right)=0\right\}$.

It follows in this case that the $\lambda_{s t}$ above are orders of coincidence of pairs of different factors or characteristic exponents of the factors.

The partial order in $\boldsymbol{Q}^{d}$, defined by $\lambda \leqslant \lambda^{\prime}$ if and only if we have ' $\leqslant$ ' coordinate wise, induces a total order in the set of characteristic exponents of a quasi-ordinary branch (see [L4, Lemma 5.6]). We relabel them in a unique form $\lambda_{1}<\lambda_{2}<\cdots<\lambda_{g}$ (where ' $<$ ' means ' $\leqslant$ ' and ' $\neq$ ').

Lemma 2.2 (cf. Proposition 1.5 of [L3] and Proposition 1.3 of [Gau]). Let $\zeta=\sum c_{\lambda} X^{\lambda}$ be a non-unit in $\boldsymbol{C}\left\{X^{1 / n}\right\}$. Then $\zeta$ is a quasi-ordinary branch if and only if there exist elements $\lambda_{i} \in(1 / k) \boldsymbol{Z}_{\geqslant 0}^{d}(1 \leqslant i \leqslant g)$ such that

(i) $\lambda_{1}<\lambda_{2}<\cdots<\lambda_{g}$ and $c_{\lambda_{i}} \neq 0$ for $1 \leqslant i \leqslant g$;

(ii) if $c_{\lambda} \neq 0$, then $\lambda$ is in the subgroup of $\boldsymbol{Q}^{d}$ given by $\boldsymbol{Z}^{d}+\sum_{\lambda_{i} \leqslant \lambda} \boldsymbol{Z} \lambda_{i}$;

(iii) $\lambda_{j}$ is not in the subgroup of $\boldsymbol{Q}^{d}$ given by $\boldsymbol{Z}^{d}+\sum_{\lambda_{i}<\lambda_{j}} \boldsymbol{Z} \lambda_{i}$ for $j=1, \ldots, g$.

If such elements exist, they are uniquely determined by $\zeta$, and they are the characteristic exponents of $\zeta$.

Remark 2.3. Lemma 2.2 gives us a canonical way of writing the terms of a quasiordinary branch,

$$
\zeta=p_{0}+p_{1}+\cdots+p_{g},
$$

where $p_{0}$ is in $\boldsymbol{C}\{X\}$ and $X^{\lambda}$ appears in $p_{i}$ with non-zero coefficient implies that $\lambda_{i} \leqslant \lambda$ and $\lambda_{i+1} \nless \lambda$.

We say that the quasi-ordinary branch $\zeta$ has well-ordered variables if the $g$-tuples $\left(\lambda_{1, i}, \ldots, \lambda_{g, i}\right)$ of $i$-coordinates of the characteristic exponents $\lambda_{1}, \ldots, \lambda_{g}$ are ordered lexicographically, i.e. we have that

$$
\left(\lambda_{1, i}, \ldots, \lambda_{g, i}\right) \geqslant_{\text {lexicographically }}\left(\lambda_{1, j}, \ldots, \lambda_{g, j}\right) \text { for } 1 \leqslant i<j \leqslant d .
$$


It is clear that, given a quasi-ordinary branch $\zeta$, we can relabel the variables $X_{1}, \ldots, X_{d}$ in order to satisfy this condition.

Definition 2.4. The quasi-ordinary branch $\zeta$ is normalized if it is given with wellordered variables, and if it happens that the first characteristic exponent is of the form $\lambda_{1}=\left(\lambda_{1,1}, 0, \ldots, 0\right)$, then we have $\lambda_{1,1}>1$.

This condition in the case of plane curves implies that the projection $(X, Y) \mapsto X$ is transversal. Lipman proved that any quasi-ordinary hypersurface is parametrized by a normalized quasi-ordinary branch $\zeta$ (see $[\mathbf{L} \mathbf{1}]$ and the appendix of $[\mathbf{G a u}]$ ).

We denote the lattice $\boldsymbol{Z}^{d}$ by $M$ and by $M_{j}$ the lattice $\boldsymbol{Z}^{d}+\sum_{\lambda_{i}<\lambda_{j+1}} \boldsymbol{Z} \lambda_{i}$ for $j=1, \ldots, g$, with the convention $\lambda_{g+1}=+\infty$. Following Lipman, we associate to the characteristic exponents sequences of lattices and integers (see [L4, p. 61]). In the planebranch case, the sequence of integers coincide with the first component of the characteristic pairs in arbitrary coordinates (see [GP2]).

Definition 2.5. The characteristic lattices are the lattices $M_{i}$ defined above. The characteristic integers are the indexes $n_{j}$ of $M_{j-1}$ in $M_{j}$ for $j=1, \ldots, g$.

We denote by $e_{i-1}=n_{i} \cdots n_{g}$ for $i=1, \ldots, g$ and we set $n_{0}:=1$.

We denote by $N$ the dual lattice of $M$ and by $N_{\boldsymbol{R}}$ the real vector space $N \otimes_{\boldsymbol{Z}} \boldsymbol{R}$ spanned by the lattice $N$. We denote by $\rho \subset N_{\boldsymbol{R}}$ the cone spanned by the dual basis of the canonical basis of $M$. The exponents of the quasi-ordinary branch $\zeta$ belong to the semigroup $\rho^{\vee} \cap M_{g}$, where $\rho^{\vee}=\left\{u \in M_{\boldsymbol{R}} /\langle u, v\rangle \geqslant 0 \forall v \in \rho\right\}$ is the dual cone of the cone $\rho$. The series $\zeta$ can be viewed as an element of the ring $C\left\{\rho^{\vee} \cap M_{g}\right\}$ of germs of holomorphic functions at the special point of the affine toric variety $\operatorname{Spec} \boldsymbol{C}\left[\rho^{\vee} \cap M_{g}\right]$ (see [GP3]). We denote the ring $\boldsymbol{C}\{X\}$ by $\boldsymbol{C}\left\{\rho^{\vee} \cap M\right\}$. The advantage of these notations is that we can define ring homomorphisms by changing the lattice or the cones, for instance, the ring extension $R=\boldsymbol{C}\left\{\rho^{\vee} \cap M\right\}[\zeta] \rightarrow \boldsymbol{C}\left\{\rho^{\vee} \cap M_{g}\right\}$ is the inclusion in the integral closure (see [GP3, Proposition 14]).

The field of fractions of $R$ is $L[\zeta]$, since $\zeta$ is algebraic over $L$.

Lemma 2.6 (cf. Lemma 5.7 of [L4]). We have the following equality:

$$
L[\zeta]=L\left[X^{\lambda_{1}}, \ldots, X^{\lambda_{g}}\right] .
$$

Remark 2.7. The integers $n_{j}$ and $e_{i}$ are the degrees of the Galois extensions,

$$
\left.\begin{array}{rlr}
e_{i} & :=\left[L[\zeta]: L\left[X^{\lambda_{1}}, \ldots, X^{\lambda_{i}}\right]\right] & \text { for } i=1, \ldots, g, \\
n_{j}:=\left[L\left[X^{\lambda_{1}}, \ldots, X^{\lambda_{j}}\right]: L\left[X^{\lambda_{1}}, \ldots, X^{\lambda_{j-1}}\right]\right] & \text { for } j=1, \ldots, g .
\end{array}\right\}
$$

In particular, we have that $\operatorname{deg} f=e_{0}=n_{1} \cdots n_{g}=n$.

It follows from Lemma 2.6 that the polynomial $f$ has all its roots in the field $L[\zeta]$ (since it is also equal to the field of fractions of $C\left\{\rho^{\vee} \cap M_{g}\right\}$ ), and thus the extension $L[\zeta]: L\left[X^{\lambda_{1}}, \ldots, X^{\lambda_{i}}\right]$ is Galois. The assertion on the degrees follows then from Definition 2.5 and Lemma 2.6 using the fact that the minimal polynomial of $X^{\lambda_{j}}$ over $L\left[X^{\lambda_{1}}, \ldots, X^{\lambda_{j-1}}\right]$ is $Y^{n_{j}}-X^{n_{j} \lambda_{j}}$. 


\section{The semigroup of a quasi-ordinary branch}

In the following sections we study the singularity $(S, o)$ defined parametrized by a quasiordinary branch $\zeta$ with $g \geqslant 1$ characteristic exponents $\lambda_{1}, \ldots, \lambda_{g}$.

If $d=1$, then $S$ is a plane branch and the set of intersection multiplicities $\left(S, S^{\prime}\right)_{0}$ of $S$ that those plane-curve germs $S^{\prime}$ not containing $S$ as a component defines the semigroup of the branch $S$. A set of generators of the semigroup is obtained from the characteristic exponents by the following formula (see $[\mathbf{Z 2}]$ ):

$$
\bar{\gamma}_{1}=n \lambda_{1}, \quad \bar{\gamma}_{j+1}=n_{j} \bar{\gamma}_{j}+n \lambda_{j+1}-n \lambda_{j} \quad \text { for } j=1, \ldots, g-1 .
$$

For $j=0, \ldots, g-1$, we expand,

$$
\begin{aligned}
\bar{\gamma}_{j+1} & =n\left(\left(n_{1}-1\right) n_{2} \cdots n_{j} \lambda_{1}+\left(n_{2}-1\right) n_{3} \cdots n_{j} \lambda_{2}+\cdots+\left(n_{j}-1\right) \lambda_{j}+\lambda_{j+1}\right) \\
& =n_{1} \cdots n_{j}\left(\left(e_{0}-e_{1}\right) \lambda_{1}+\left(e_{1}-e_{2}\right) \lambda_{2}+\cdots+\left(e_{j-1}-e_{j}\right) \lambda_{j}+e_{j} \lambda_{j+1}\right)
\end{aligned}
$$

We denote $(1 / n) \bar{\gamma}_{i}$ by $\gamma_{i}$ for $i=1, \ldots, g$. We have

$$
\gamma_{1}=\lambda_{1}, \quad \gamma_{j+1}=n_{j} \gamma_{j}+\lambda_{j+1}-\lambda_{j} \quad \text { for } j=1, \ldots, g-1 .
$$

Recall that if $q \in C\{X\}[Y]$ defines a germ of curve $S^{\prime}$ at the origin, the intersection multiplicity $\left(S, S^{\prime}\right)_{0}=\operatorname{dim}_{C} C\{X, Y\} /(f, q)$ coincides with the order in $X$ of the resultant $\operatorname{Res}_{Y}(f, q)$ of the polynomials $f$ and $q$ with respect to $Y$.

Proposition 3.1. Let $q \in C\left\{\rho^{\vee} \cap M\right\}[Y]$ be any irreducible quasi-ordinary polynomial of degree $n_{0} n_{1} \cdots n_{j}$ for $0 \leqslant j \leqslant g-1$. The following are equivalent.

(1) The polynomial $q$ has order of coincidence $\lambda_{j+1}$ with $f$.

(2) $\operatorname{Res}_{Y}(f, q)=X^{\bar{\gamma}_{j+1}} \varepsilon_{j}$ for a unit $\varepsilon_{j} \in C\left\{\rho^{\vee} \cap M\right\}$.

(3) $q(\zeta)=X^{\gamma_{j+1}} \epsilon_{j}$ for a unit $\epsilon_{j} \in C\left\{\rho^{\vee} \cap M_{g}\right\}$.

Proof. The result is trivial for $j=0$. Suppose first that $q$ is an irreducible quasi-ordinary polynomial of degree $\geqslant 1$ comparable with $f$ and having order of coincidence $\alpha$. Since the roots $\left\{\zeta^{(k)}\right\}_{k=1}^{n}$ of $f$ are a complete set of conjugates over $L$ and the polynomial $q$ has its coefficients on this ring, the series $\left\{q\left(\zeta^{(k)}\right)\right\}_{k=1}^{n}$ are a complete set of conjugates over $L$. Thus we have

$$
\mathcal{N}\left(\operatorname{Res}_{Y}(f, q)\right)=\mathcal{N}\left(\prod_{k=1}^{n} q\left(\zeta^{(k)}\right)\right)=\operatorname{deg} f \mathcal{N}(q(\zeta)) .
$$

We deduce from (3.4) the equivalence between assertions (2) and (3). If $\tau$ is any root of the irreducible polynomial $q$, we deduce by symmetry from (3.4) that

$$
\mathcal{N}\left(\operatorname{Res}_{Y}(f, q)\right)=\operatorname{deg} q \mathcal{N}(f(\tau)) .
$$

Take a root $\zeta^{(k)}$ of $f$ such that $\zeta^{(k)}-\tau=X^{\alpha} \cdot$ unit. By definition of the order of coincidence, the biggest characteristic exponent of $\tau$ that is less than $\alpha$, if it exists, is a 
characteristic exponent $\lambda_{i}$ of $\zeta^{(k)}$. The roots of $f$ verifying this property are obtained from $\zeta^{(k)}$ by the action of the Galois group of the extension $L\left[X^{\lambda_{1}}, \ldots, X^{\lambda_{j}}\right] \subset L[\zeta]$ by Remark 2.7 and conversely thus

$$
\#\left\{\operatorname{roots} \zeta^{(l)} \text { of } f \text { such that } \zeta^{(l)}-\tau=X^{\alpha} \cdot \text { unit }\right\}=\left[L[\zeta]: L\left[X^{\lambda_{1}}, \ldots, X^{\lambda_{i}}\right]\right]=e_{i} \text {. }
$$

Analogously, we obtain that

$$
\#\left\{\operatorname{roots} \zeta^{(l)} \text { of } f \text { such that } \zeta^{(l)}-\tau=X^{\lambda_{k}} \text {.unit }\right\}=e_{k-1}-e_{k} \quad \text { for } k=1, \ldots, i \text {. }
$$

By (3.4) and (3.5), this implies that $\operatorname{Res}_{Y}(f, q)=X^{\gamma} \cdot$ unit, where

$$
\gamma=\operatorname{deg} q\left(\left(e_{0}-e_{1}\right) \lambda_{1}+\left(e_{1}-e_{2}\right) \lambda_{2}+\cdots+\left(e_{i-1}-e_{i}\right) \lambda_{i}+e_{i} \alpha\right) .
$$

If $\alpha=\lambda_{j+1}$, then we have that $i=j$ and $\gamma=\bar{\gamma}_{j+1}$ by equation (3.2) and the equality $\operatorname{deg} q=n_{1} \cdots n_{j}$.

Conversely, if $1 \leqslant i<j$ or if $\alpha$ is less than or equal to any characteristic exponent of $\tau$, we deduce from formulae (3.2) and (3.7) that $\alpha \geqslant \lambda_{j+1}$. This implies that $\lambda_{j}$ is less than $\alpha$ and it is also a characteristic exponent of $\tau$; a contradiction. If $j<i$, we obtain from (3.2) and (3.7) that $\gamma>\bar{\gamma}_{j+1}$. Therefore, $\gamma=\bar{\gamma}_{j+1}$ implies that $i=j$ and $\alpha=\lambda_{j+1}$.

Remark 3.2. With the previous notations, the leading exponent $\delta$ of the discriminant of $f$ (which is defined by $\Delta_{Y} f=X^{\delta} \varepsilon$, for $\varepsilon$ a unit) is equal to $\delta=\sum_{k=1}^{g}\left(e_{k-1}-e_{k}\right) \lambda_{k}$. The proof follows using equation (3.6) for the roots of $f$ and the relation $\Delta_{Y} f=$ $\prod_{i \neq j}\left(\zeta^{(i)}-\zeta^{(j)}\right)$.

We associate to the characteristic monomials of the quasi-ordinary branch $\zeta$ the sequence of semigroups

$$
\Gamma_{j}=\rho^{\vee} \cap M+\gamma_{1} \boldsymbol{Z}_{\geqslant 0}+\cdots+\gamma_{g} \boldsymbol{Z}_{\geqslant 0} \quad \text { for } j=0, \ldots, g .
$$

We denote $n \Gamma_{j}$ by $\bar{\Gamma}_{j}$ for $j=0, \ldots, g$. If $d=1$, the semigroup $\bar{\Gamma}_{g} \subset \boldsymbol{Z}_{\geqslant 0}$ is the classical semigroup of the plane branch $S$.

\section{Lemma 3.3 (cf. Chapter I, Lemma 2.2.1 of [T] in the plane-branch case and [GP2]).}

(1) The sub-lattice of $M_{g}$ generated by $\Gamma_{j}$ is equal to $M_{j}$ for $0 \leqslant j \leqslant g$.

(2) The order of the image of $\gamma_{j}$ in the group $M_{j} / M_{j-1}$ is equal to $n_{j}$ for $j=1, \ldots, g$.

(3) We have that $\gamma_{j}>n_{j-1} \gamma_{j-1}$ for $j=2, \ldots, g$.

(4) If a vector $u_{j} \in \rho^{\vee} \cap M_{j}$, then we have $u_{j}+n_{j} \gamma_{j} \in \Gamma_{j}$.

(5) The vector $n_{j} \gamma_{j}$ belongs to the semigroup $\Gamma_{j-1}$ for $j=1, \ldots, g$. Moreover, we have a unique relation

$$
n_{j} \gamma_{j}=\alpha^{(j)}+l_{1}^{(j)} \gamma_{1}+\cdots+l_{j-1}^{(j)} \gamma_{j-1}
$$

such that $0 \leqslant l_{i}^{(j)} \leqslant n_{i}-1$ and $\alpha^{(j)} \in M_{0}$ for $j=1, \ldots, g$. 
Proof. The first assertion follows from (3.3). The second assertion follows from the definition of the integers $n_{i}$ and the fact that $\gamma_{i}=\lambda_{i} \bmod M_{i-1}$ for $i=1, \ldots, g$. We deduce the third from (3.3) and the inequality

$$
\begin{aligned}
n_{j} \gamma_{j}-n_{j-1} \gamma_{j-1} & =n_{j-1}\left(n_{j}-1\right) \gamma_{j-1}+n_{j}\left(\lambda_{j}-\lambda_{j-1}\right) \\
& >\left(n_{j}-1\right)\left(n_{j-1} \gamma_{j-1}+\lambda_{j}-\lambda_{j-1}\right) \\
& =\left(n_{j}-1\right) \gamma_{j} .
\end{aligned}
$$

Assertion (4) is easy for $j=1$. We suppose it is true for $j-1 \geqslant 1$. A vector $u_{j} \in \rho^{\vee} \cap M_{j}$ is of the form $u_{j}=\alpha_{j} \gamma_{j}+u_{j}^{\prime}$ for a unique $0 \leqslant \alpha_{j}<n_{j}$ and $u_{j}^{\prime} \in M_{j-1}$. By (3.9), the vector $u_{j-1}:=u_{j}^{\prime}+n_{j} \gamma_{j}-n_{j-1} \gamma_{j-1}$ belongs to $\rho^{\vee} \cap M_{j-1}$. By induction hypothesis, the vector $u_{j-1}+n_{j-1} \gamma_{j-1}=u_{j}^{\prime}+n_{j} \gamma_{j}$ is in the semigroup $\Gamma_{j-1}$, and hence the vector $u_{j}+n_{j} \gamma_{j}=\alpha_{j} \gamma_{j}+u_{j}^{\prime}+n_{j} \gamma_{j}$ belongs to the semigroup $\Gamma_{j}$.

We deduce from (3.3) that $n_{j} \gamma_{j}=n_{j} n_{j-1} \gamma_{j-1}+n_{j}\left(\lambda_{j}-\lambda_{j-1}\right)$. By assertions (1) and 2, the vector $n_{j}\left(\lambda_{j}-\lambda_{j-1}\right)$ is in the lattice $M_{j-1}$ and, by Lemma 2.2 , it belongs to $\rho^{\vee}$. Now we apply (4) to obtain the first assertion of (5). The existence of relations of the form (3.8) follows by induction on $g$ using Euclidean division: for $g=1$ it is clear. We suppose true for $g-1$. Then, by induction, we have the required relations for $j=1, \ldots, g-1$. We have proved that $n_{j} \gamma_{g} \in \Gamma_{g}$, and thus we have a relation,

$$
n_{j} \gamma_{g}=\alpha_{0}+l_{1} \gamma_{1}+\cdots+l_{g-1} \gamma_{g-1} .
$$

We divide the non-negative integer $l_{g-1}$ by $n_{g-1}$ and we obtain $l_{g-1}=k n_{g-1}+l_{g-1}^{(g)}$ with $0 \leqslant l_{g-1}^{(g)} \leqslant n_{g-1}-1$. Then we substitute $l_{g-1} \gamma_{g-1}$ by

$$
l_{g-1}^{(g)} \gamma_{g-1}+k\left(\alpha^{(g-1)}+\sum_{i=1}^{g-2} l_{i}^{(g-1)} \gamma_{i}\right)
$$

in (3.10) and we obtain a formula of the same type where $l_{g-1}=l_{g-1}^{(g)}$. The required expansion is obtained by iterating this procedure. The unicity follows from (2).

We say that a set of generators of a semigroup is minimal if none of the generators belongs to the semigroup spanned by the others.

Lemma 3.4. The semigrup $\Gamma_{g}$ has a unique minimal set of generators. If $\zeta$ is normalized, this set is the canonical basis of $M_{0}$ union $\left\{\gamma_{1}, \ldots, \gamma_{g}\right\}$.

Proof. Since the semigroup $\Gamma_{\zeta}$ is contained in the cone with vertex $\rho^{\vee}$, we can use Lemmas 3.6 and 3.5 of [Ew, Chapter $\mathrm{V}]$ to prove that $\Gamma_{\zeta}$ has a minimal set of generators, which is unique. We show that the canonical basis vectors and $\gamma_{1}, \ldots, \gamma_{g}$ are a minimal set of generators of $\Gamma_{\zeta}$. The condition of being normalized implies that the canonical basis vectors are the first elements of the semigroup $\Gamma_{\zeta}$ appearing on the edges of the cone $\rho^{\vee}$, and hence we cannot eliminate any of them while preserving the semigroup. They generate the subsemigroup $\Gamma_{0}=\rho^{\vee} \cap M_{0}$ of $\Gamma_{g}$. If we have a relation of the form $\gamma_{k}=u+\sum a_{j} \gamma_{j}$, with $u \in \Gamma_{0}, a_{j} \in \boldsymbol{Z}_{\geqslant 0}$ and $a_{k}=0$, then assertion (3) of Lemma 3.3 implies that $a_{j}=0$ for $j>k$. The relation obtained contradicts assertion (2) of Lemma 3.3. 
Remark 3.5. Properties (1), (2), (3) and (5) of Lemma 3.3 characterize those semigroups $\Lambda$ of the form

$$
\Lambda=\rho^{\vee} \cap M_{0}+\gamma_{1} \boldsymbol{Z}_{\geqslant 0}+\cdots+\gamma_{g} \boldsymbol{Z}_{\geqslant 0} \quad \text { for } \gamma_{i} \in \rho^{\vee} \cap M_{\boldsymbol{Q}}, \quad i=1, \ldots, g,
$$

which are associated to the characteristic monomials of a quasi-ordinary branch.

Proof. In this proof we denote by $M_{j}$ the lattice $M_{0}+\sum_{i=1}^{j} \gamma_{i} \boldsymbol{Z}$, by $\Gamma_{j}$ the semigroups $\rho^{\vee} \cap M_{0}+\sum_{i=1}^{j} \gamma_{i} \boldsymbol{Z}_{\geqslant 0}$ for $i=0, \ldots g$, and by $n_{i}$ the order of $\gamma_{i}$ modulo $M_{i-1}$ for $i=1, \ldots, g$. We define vectors in $M_{Q}: \alpha_{1}:=\gamma_{1}$ and $\alpha_{j+1}:=\gamma_{j+1}-\gamma_{j}+\alpha_{j}$ for $j=1, \ldots, g-1$. It follows by property (3) and induction that $\alpha_{j+1}$ is greater than $\alpha_{j}$ and belongs to the semigroup $\rho^{\vee} \cap M_{g}$ for $j=1, \ldots, g-1$. By Lemma 2.2, the series $\zeta:=X^{\alpha_{1}}+\cdots+X^{\alpha_{g}}$ is a quasi-ordinary branch with minimal polynomial in $C\left\{\rho^{\vee} \cap M_{0}\right\}[Y]$, with characteristic exponents $\alpha_{i}$ for $i=1, \ldots, g$ and with the previously defined characteristic integers $n_{1}, \ldots, n_{g}$ and lattices $M_{1}, \ldots, M_{g}$. It follows that $\Lambda=\Gamma_{g}$.

\subsection{Semigroup and Newton polyhedra}

Newton polyhedra are used in this section to characterize the semigroup associated to a quasi-ordinary branch.

The Newton polyhedron of a series $0 \neq \phi=\sum c_{a} X^{a} \in C\left\{\rho^{\vee} \cap M\right\}$ is the convex hull of the set $\bigcup_{c_{a} \neq 0} a+\rho^{\vee}$. We define the Newton polyhedron of $0 \neq \phi \in C\left\{\rho^{\vee} \cap M^{\prime}\right\}$ for $M^{\prime}$ a lattice containing $M$ in the same way. It follows that the Newton polyhedron does not change after a lattice extension. The face determined by $\eta \in \rho$ on the Newton polyhedron $\mathcal{N}(\phi)$ is the set $\left\{v \in \mathcal{N}(\phi) /\langle\eta, v\rangle=\inf _{v^{\prime} \in \mathcal{N}(\phi)}\left\langle\eta, v^{\prime}\right\rangle\right\}$. All faces of the polyhedron $\mathcal{N}(\phi)$ can be recovered in this way; in particular, the compact faces are determined by the vectors $\eta$ in the interior of $\rho$. The cone $\sigma(\mathcal{F}) \subset \rho$ associated to the face $\mathcal{F}$ of the polyhedron $\mathcal{N}(\phi)$ is

$$
\sigma(\mathcal{F}):=\left\{\eta \in \rho / \inf _{v^{\prime} \in \mathcal{N}(\phi)}\left\langle\eta, v^{\prime}\right\rangle=\langle\eta, v\rangle, \forall v \in \mathcal{F}\right\}
$$

The set of cones $\sigma(\mathcal{F})$, for $\mathcal{F}$ running through the set of faces of the polyhedron $\mathcal{N}(\phi)$, is called the dual Newton diagram of $\phi$. The Newton principal part $\phi_{\mid \mathcal{N}}$ of $\phi$ is the sum of those terms of $\phi$ having exponents lying on the compact faces of its Newton polyhedron (see $[\mathbf{K o u}]$ for the terminology).

Any non-zero element of the ring $R=C\left\{\rho^{\vee} \cap M\right\}[\zeta]$ is of the form $h(\zeta)$ for a polynomial $h \in \boldsymbol{C}\left\{\rho^{\vee} \cap M\right\}[Y]$ of degree less than $n$. The following proposition (see [GP2]) is a generalization of a result of plane branches (see [Z2, Chapter II, Theorem 3.9]).

We denote by $q_{i}$ the minimal polynomial of the truncation $p_{0}+p_{1}+\cdots+p_{i}$ of the parametrization of $f$ for $i=0, \ldots, g$ (see Remark 2.7). The polynomials $q_{i}$ are quasiordinary by Lemma 2.2 and $\operatorname{deg} q_{i}=n_{0} \cdots n_{i}$ for $i=0, \ldots, g$.

Theorem 3.6. If $\operatorname{deg} h<n_{0} \cdots n_{j}$, then the Newton principal part of $h(\zeta)$ belongs to the ring $\boldsymbol{C}\left[\Gamma_{j}\right]$ for $j=0, \ldots, g$. 
Proof. We show the result by induction. For $j=0$, if $\operatorname{deg} h<n_{0}=1$, the result is trivial, since $\Gamma_{0}=\rho^{\vee} \cap M$. We suppose the result holds for degrees less than $n_{0} \cdots n_{j-1}$. If $\operatorname{deg} h<n_{1} \cdots n_{j}$, using Euclidean division several times we can expand the polynomial $h$ in a unique way: the $q_{j-1}$-adic expansion of $h$,

$$
h=a_{0}+a_{1} q_{j-1}+\cdots+a_{s} q_{j-1}^{s},
$$

where $a_{k}$ are polynomials in $C\left\{\rho^{\vee} \cap M\right\}[Y]$ of degree less than $n_{1} \cdots n_{j-1}=\operatorname{deg} q_{j-1}$ for $k=0, \ldots, s$ and $0 \leqslant s<n_{j}$. The Newton principal part of $\left(a_{k} q_{j-1}^{k}\right)(\zeta)$ is the product of the Newton principal parts of the factors. We have that $\left(q_{j-1}^{k}(\zeta)\right)_{\mid \mathcal{N}}=X^{k \gamma_{j}}$ and, by induction hypothesis, $a_{k}(\zeta)_{\mid \mathcal{N}}$ belongs to the ring $\boldsymbol{C}\left[\Gamma_{j-1}\right]$. The exponents of the Newton principal part of $\left(a_{k} q_{j-1}^{k}\right)(\zeta)$ belong to the set $k \gamma_{j}+M_{j-1}$ for $k=0, \ldots, s$ and, by Lemma 3.3, these sets are disjoint since the order $n_{j}$ of $\gamma_{j}$ in the group $M_{j} / M_{j-1}$ is greater than $s$. It follows that the terms of the Newton principal parts of $\left(a_{k} q_{j-1}^{k}\right)(\zeta)$ cannot cancel each other, and thus the polynomial $h(\zeta)_{\mid \mathcal{N}}$ is a sum of the terms of the Newton principal parts of $\left(a_{k} q_{j-1}^{k}\right)(\zeta)$ for $k=0, \ldots, s$.

Corollary 3.7. The Newton principal part of $\operatorname{Res}_{Y}(f, h)$, for those polynomials $h$ in $\boldsymbol{C}\{X\}[Y]$ that are not divisible by $(f)$, runs through the elements of the ring $\boldsymbol{C}\left[\bar{\Gamma}_{g}\right]$.

The set of vertices of Newton polyhedra of $\operatorname{Res}_{Y}(f, h)$ (respectively, of $h(\zeta)$ ) for $h \in(\boldsymbol{C}\{X\}[Y] \backslash(f))$ is a semigroup with respect to the addition which is isomorphic to $\Gamma_{g}$.

Proof. The proof is a consequence of (3.4) and of Theorem 3.6.

This corollary gives the analogy with the classical definition of the semigroup of a plane branch using the canonical valuation of the integral closure of the ring $R$. We say that the semigroup $\Gamma_{g}$ is associated to the quasi-ordinary branch $\zeta$ and we denote it also by $\Gamma_{\zeta}$.

Remark 3.8. An alternate way of defining the semigroup $\Gamma_{\zeta}$ is given by Popescu-Pampu in [PP2]: he introduces first the set $\mathcal{C}_{f}$ of functions $h \in \boldsymbol{C}\{X\}[Y] \backslash(f)$ such that the Newton polyhedron of $h(\zeta)$ has only one vertex $\gamma_{h}$, then he defines the semigroup by $\Gamma_{\zeta}=\left\{\gamma_{h} / h \in \mathcal{C}_{f}\right\}$.

Remark 3.9. Any polynomial $h \in C\left\{\rho^{\vee} \cap M\right\}[Y]$ can be written in a unique way as

$$
h=\sum c_{l_{1}, \ldots, l_{g+1}} q_{0}^{l_{1}} q_{1}^{l_{2}} \cdots q_{g}^{l_{g+1}},
$$

with $c_{l_{1}, \ldots, l_{g+1}} \in C\left\{\rho^{\vee} \cap M\right\}, 0 \leqslant l_{k} \leqslant n_{k}-1$ for $k=1, \ldots, g$ and $l_{g+1} \in Z_{\geqslant 0}$.

This follows by computing the $q_{g}$ expansion of $h$ first and then the $q_{g-1}$ expansion of the coefficients and iterating the procedure (see $[\mathbf{A} 3]$ in the case of plane branches). By using Lemma 3.3, we deduce the following fact (see [PP2]).

Remark 3.10. The exponents of the Newton principal part of the terms

$$
\left(c_{l_{1}, \ldots, l_{g+1}} q_{0}^{l_{1}} q_{1}^{l_{2}} \cdots q_{g-1}^{l_{g}}\right)(\zeta)
$$

cannot cancel each other. 


\section{Semi-roots and approximate roots}

In the plane-branch case, several authors have studied the properties of those curves $S^{\prime}$ such that the intersection multiplicity with $S$ at the origin belongs to the unique minimal set of generators of the semigroup of the branch (see $[\mathbf{Z 2}]$ ). In $[\mathbf{L J 1}]$, Lejeune introduced the notion of curves of maximal contact of higher genus with a given plane-curve germ for curves defined over a field of arbitrary characteristic in terms of the resolution (see [LJ2]). If the characteristic is zero, it turns out that both notions are equivalent (see [Ca]). If the projection $(X, Y)$ is transversal, we can study these curves by means of the minimal polynomials of suitable truncations of the roots of $f$. When we do this with respect to an arbitrary projection, the curves we obtain provide a non-necessarily minimal set of generators of the semigroup of the branch $S$. We call them semi-roots, following the terminology of Abhyankar and Popescu-Pampu (see $[\mathbf{A 3}, \mathbf{P P 1}]$ ). They are also called pseudo-roots by Gwoździewicz and Ploski (see $[\mathbf{G}-\mathbf{P}]$ ). Approximate roots are used by Abhyankar and Moh (see [A-M1, A-M2]) to obtain several results on the local and global geometry of plane curves. We show that the approximate roots of $f$ of degrees $e_{j}$ for $j=0, \ldots, g$ are semi-roots of $f$ (see $[\mathbf{G P 2}]$ ). We follow the approach of the planecurve case given by Gwoździewicz and Ploski (see $[\mathbf{G}-\mathbf{P}]$ ) and by Popescu-Pampu in the survey $[\mathbf{P P 1}]$.

Definition 4.1. A $j$-semi-root of $f$ is an irreducible quasi-ordinary polynomial in $\boldsymbol{C}\left\{\rho^{\vee} \cap M\right\}[Y]$ of degree $n_{0} \cdots n_{j}$ that has order of coincidence equal to $\lambda_{j+1}$ with $f$ for $j=0, \ldots, g$.

The minimal polynomials of the quasi-ordinary branches $p_{0}+\cdots+p_{j}$ obtained by truncating $\zeta$ in Remark 2.3 are $j$-semi-roots of $f$ for $j=0, \ldots, g$.

Proposition 4.2. Let $q \in C\left\{\rho^{\vee} \cap M\right\}[Y]$ be a monic polynomial of degree $n_{0} \cdots n_{j}$. Then $q$ is a $j$-semi-root of $f$ if and only if $q(\zeta)=X^{\gamma_{j+1}} \varepsilon_{j}$ for a unit $\varepsilon_{j}$ in $\boldsymbol{C}\left\{\rho^{\vee} \cap M_{g}\right\}$.

Proof. We have to show that if $q(\zeta)$ is of the form $X^{\gamma_{j+1}} \varepsilon_{j}$ for a unit $\varepsilon_{j}$, then $q$ is necessarily an irreducible quasi-ordinary polynomial with order of coincidence $\lambda_{j+1}$ with $f$.

The polynomial $q$ is irreducible. Suppose that it is equal to the product $q_{1} q_{2}$ of polynomials of degrees less than $n_{1} \cdots n_{j}$. Then we have that $q(\zeta)=q_{1}(\zeta) q_{2}(\zeta)=X^{\gamma_{j+1}} \varepsilon_{j+1}$ for a unit $\varepsilon_{j+1} \in C\left\{\rho^{\vee} \cap M_{g}\right\}$. The Newton polyhedron of $q(\zeta)$ has only one vertex and since this polyhedron is equal to the Minkowski sum $\sum_{i=1,2} \mathcal{N}\left(q_{i}(\zeta)\right)$ and it follows that each polyhedron $\mathcal{N}\left(q_{i}(\zeta)\right)$ has only one vertex $u_{i}$. We deduce that $q_{i}(\zeta)=X^{u_{i}} \epsilon_{i}$ for $\epsilon_{i}$ a unit in $\boldsymbol{C}\left\{\rho^{\vee} \cap M_{g}\right\}$. Then Proposition 3.6 implies that $\gamma_{j+1}=u_{1}+u_{2}$ belongs to $\Gamma_{j}$, contradicting Lemma 3.3.

By Proposition 3.1, the result follows by proving that the polynomial $q$ is quasiordinary, i.e. that the Newton polyhedron of the discriminant $\Delta_{Y} q$ has only one vertex. The cone associated to a vertex $v$ of the polyhedron $\mathcal{N}\left(\Delta_{Y} q\right)$,

$$
\sigma_{v}=\left\{u \in \rho / \inf _{v^{\prime} \in \mathcal{N}\left(\Delta_{Y} q\right)}\left\langle u, v^{\prime}\right\rangle=\langle u, v\rangle\right\}
$$

is of dimension $d$. Let $\sigma \subset \sigma_{v}$ be any cone generated by a basis of the lattice $N$ (see [Ew] for its existence). The dual cone $\sigma^{\vee}$ of $\sigma$ is generated by a basis of the dual lattice $M$ 
and contains $\rho^{\vee}$. We have the local ring extension $\boldsymbol{C}\left\{\rho^{\vee} \cap M\right\} \hookrightarrow \boldsymbol{C}\left\{\sigma^{\vee} \cap M\right\}$ defined by the semigroup inclusion $\rho^{\vee} \cap M \hookrightarrow \sigma^{\vee} \cap M$. We obtain a similar extension by replacing the lattice $M$ by $M_{g}$. The ring $C\left\{\sigma^{\vee} \cap M\right\}$ is isomorphic to the $\operatorname{ring} C\left\{T_{1}, \ldots, T_{d}\right\}$, the isomorphism maps $T_{i}$ to $X^{t_{i}}$ for $t_{1}, \ldots, t_{d}$ the basis of $M$ that spans $\sigma^{\vee}$ as a cone.

We denote an element $\phi$ in $C\left\{\rho^{\vee} \cap M\right\}$ (respectively, in $C\left\{\rho^{\vee} \cap M\right\}[Y]$ ) viewed as an element of $\boldsymbol{C}\left\{\sigma^{\vee} \cap M\right\}$ (respectively, of $\boldsymbol{C}\left\{\sigma^{\vee} \cap M\right\}[Y]$ ) by $\phi_{\sigma}$. The polynomial $f_{\sigma}$ is quasi-ordinary: its roots $\zeta_{\sigma}^{(i)}$, for $\zeta^{(i)}$ running through the roots of $f$, are conjugated quasi-ordinary branches by Lemma 2.2 , since the characteristic monomials of $\zeta_{\sigma}$ are the images of the characteristic monomials of $\zeta$ by the local ring extension $\boldsymbol{C}\left\{\rho^{\vee} \cap M_{g}\right\} \hookrightarrow \boldsymbol{C}\left\{\sigma^{\vee} \cap M_{g}\right\}$, and the characteristic lattices coincide for $\zeta$ and $\zeta_{\sigma}$.

The polynomial $q_{\sigma}$ is quasi-ordinary, since its Newton polyhedron is equal to the Minkowski sum $\mathcal{N}\left(\Delta_{Y} q\right)+\sigma^{\vee}$ and it has a unique vertex $v$, since $\sigma \subset \sigma_{v}$. By the argument given in the first paragraph of this proof, the polynomial $q_{\sigma}$ is irreducible and it follows from Proposition 3.1 that it is a $j$-semi-root of $f_{\sigma}$. The vertex $v$ is determined by the first $j$ characteristic exponents of $f$ and the associated characteristic integers (more precisely, we have $v=\sum_{k=1}^{j}\left(e_{k-1}-e_{k}\right) \lambda_{k}$ by Remark 3.2). This implies that the polyhedron $\mathcal{N}\left(\Delta_{Y}(q)\right)$ has only one vertex $v$ and $q \in C\left\{\rho^{\vee} \cap M\right\}[Y]$ is a quasi-ordinary polynomial.

Let $A$ be a ring containing $\boldsymbol{Q}$ as a subring. If $p$ is any monic polynomial and $k$ divides the degree of $p$, there is a unique monic polynomial $r$ in $A[Y]$ of degree $\operatorname{deg}(p) / k$ such that $\operatorname{deg}\left(p-r^{k}\right)<\operatorname{deg}(p)-\operatorname{deg}(p) / k$. We denote this polynomial by $\sqrt[k]{p}$. For instance, if $p=Y^{n}-a_{1} Y^{n-1}+\cdots+a_{0}$, we have that $\sqrt[n]{p}=Y-a_{1} / n$. If $k=k_{1} k_{2}$ divides $\operatorname{deg} p$, then we have that $\sqrt[k]{p}=\sqrt[k_{1}]{k_{2}} \sqrt{p}$ (see [PP1, Proposition 3.3]). If $q \in A[Y]$ is any monic polynomial of degree $\operatorname{deg}(p) / k$, we expand, $p=q^{k}+a_{1} q^{k-1}+\cdots+a_{0}$, with polynomials $a_{i} \in A[Y]$ of degree less than $\operatorname{deg}(p) / k$. The map $\mathcal{T}_{p}$ between the set of monic polynomials of degree $\operatorname{deg}(p) / k$ defined by

$$
\mathcal{T}_{p}(q)=q+\frac{1}{k} a_{1}
$$

is called the $k$-Tschirnhausen operator. It is shown that the $k$-approximate roots can be computed by iterating the $k$-Tschirnhausen operator, i.e

$$
\sqrt[k]{p}=\overbrace{\mathcal{T}_{p} \circ \cdots \circ \mathcal{T}_{p}}^{\operatorname{deg}(p) / k}(q),
$$

where $q$ is any monic polynomial of $\operatorname{degree} \operatorname{deg}(p) / k$ (see [PP1, Proposition 6.3]).

Proposition 4.3. The $e_{j}$-approximate roots of $f$ are $j$-semi-roots of $f$.

Proof. This is trivial for $j=0$ and is also trivial for $j=g$, since we have that $\sqrt[1]{f}=f$ satisfies the condition (for $\lambda_{g+1}=\infty$ ). Suppose the result is true for $1<j<g$. We show that it is true for $j-1$. We have that $e_{j-1}=n_{j} e_{j}$ and $\sqrt[e_{j}-1]{f}=\sqrt[n_{j}]{\sqrt[e_{j}]{f}}$. If we set $p=\sqrt[e_{j}]{f}$, we obtain that

$$
\sqrt[e_{j}-1]{f}=\overbrace{\mathcal{T}_{p} \circ \cdots \circ \mathcal{T}_{p}}^{n_{j}}(q),
$$

where $q$ is any monic polynomial of degree $n_{1} \cdots n_{j-1}$. 
It is sufficient to prove that if $q$ is a $(j-1)$-semi-root, the polynomial $\mathcal{T}_{p}(q)$ is a $(j-1)$-semi-root. We expand, $p=q^{n_{j}}+a_{1} q^{n_{j}-1}+\cdots+a_{0}$, with $a_{i} \in C\left\{\rho^{\vee} \cap M\right\}[Y]$ polynomials of degree less than $\operatorname{deg} q$. By the induction hypothesis, $p$ is a $j$-semi-root of $f$, and thus $\mathcal{N}\left(a_{1}(\zeta) q^{n_{j}-1}(\zeta)\right) \subset \mathcal{N}(p(\zeta))=\gamma_{j+1}+\rho^{\vee}$. It means that if $u$ is any vertex of $\mathcal{N}\left(a_{1}(\zeta)\right)$, then $u \geqslant \gamma_{j+1}-\left(n_{j}-1\right) \gamma_{j}$ is greater than $\gamma_{j}$ by Lemma 3.3. It follows that $q+\left(1 / n_{j}\right) a_{1}$ is a $(j-1)$-semi-root of $f$ by Proposition 4.2 .

\section{Invariance of the semigroup and inversion formulae}

In this section we prove that the semigroups associated to a quasi-ordinary branch $\zeta$ and the normalized quasi-ordinary branch obtained from $\zeta$ by Lipman's inversion lemma are isomorphic. We obtain from this another proof of the inversion formulae relating their characteristic monomials. We show then that this semigroup is a complete topological invariant of the embedded topological type of the hypersurface parametrized by $\zeta$, as characterized by the work of Gau and Lipman.

If $\zeta$ is of the form $\zeta=X_{1}^{k / n} H\left(X_{1}^{1 / n}, \ldots, X_{d}^{1 / n}\right)$, with $H(0) \neq 0$ and with minimal polynomial $f \in C\{X\}[Y]$, the order of the series $f\left(X_{1}, 0, \ldots, 0 ; 0\right)$ is equal to $k$ (for $n=\operatorname{deg} f)$. The Weierstrass polynomial $f^{\prime} \in C\left\{Y, X_{2}, \ldots, X_{d}\right\}\left[X_{1}\right]$ associated to $f$ defines the same hypersurface germ. The inversion lemma of Lipman (see [L1, Lemma 2.3] or the appendix of $[\mathbf{G a u}]$ ) guarantees that $f^{\prime}$ is a quasi-ordinary polynomial and, if $\zeta$ is not normalized, any root $\tau$ of $f^{\prime}$ is. This lemma is a generalization of the classical inversion lemma of plane branches (see $[\mathbf{Z 1}, \mathbf{A 2}]$ ).

A root $\tau$ of $f^{\prime}$ can be obtained as follows. Let $F$ be a unit such that $F^{k}=H$. The series $\zeta$ is equal to $\zeta=\zeta_{0}^{k}$, where $\zeta_{0}=X_{1}^{1 / n} F\left(X_{1}^{1 / n}, \ldots, X_{d}^{1 / n}\right)$. The series $Y^{1 / k}-\zeta_{0}$ is of order one in $X_{1}^{1 / n}$ and, by the Weierstrass preparation theorem, there exists a unit $\varepsilon$ such that

$$
\varepsilon\left(Y^{1 / k}-\zeta_{0}\right)=X_{1}^{1 / n}-\tau_{0},
$$

where $\tau_{0}$ is of the form $\tau_{0}=Y^{1 / k} F^{\prime}\left(Y^{1 / k}, X_{2}^{1 / n}, \ldots, X_{d}^{1 / n}\right)$ for a unit $F^{\prime}$.

We build from (5.1) an isomorphism,

$$
C\left\{X_{1}^{1 / n}, \ldots, X_{d}^{1 / n}\right\} \rightarrow C\left\{Y^{1 / k}, X_{2}^{1 / n}, \ldots, X_{d}^{1 / n}\right\}
$$

which maps $X_{1}^{1 / n} \mapsto \tau_{0}, X_{i}^{1 / n} \mapsto X_{i}^{1 / n}$ for $i=2, \ldots, d$, and such that the inverse image of $Y^{1 / k}$ (respectively, of $Y$ ) is equal to $\zeta_{0}$ (respectively, to $\zeta_{0}^{k}=\zeta$ ). Since $\zeta$ is a root of $f$, we have $f^{\prime}\left(\zeta, X_{2}, \ldots, X_{d} ; X_{1}\right)=0$, and thus its image by $(5.2)$ is $f^{\prime}\left(Y, X_{2}, \ldots, X_{d}, \tau_{0}^{n}\right)=0$. Therefore, $\tau:=\tau_{0}^{n}$ is a root of $f^{\prime}$.

Moreover, we have a commutative diagram

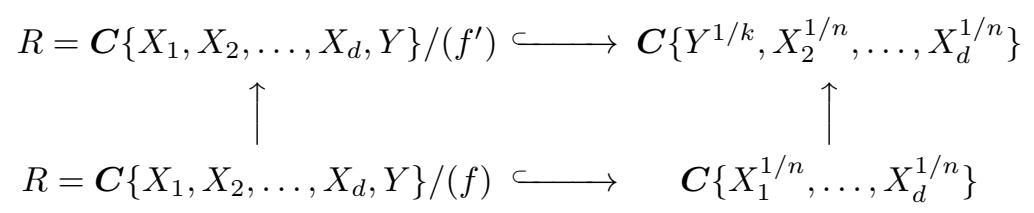

where the horizontal arrows are the ring extensions defined by $\tau$ and $\zeta$ and the left vertical arrow is the identity and the right vertical arrow is the isomorphism (5.2). 
If the Newton polyhedron of $0 \neq \phi \in\left\{X_{1}^{1 / n}, \ldots, X_{d}^{1 / n}\right\}$ has only one vertex, the same happens for the Newton polyhedron of the image of $\phi$ by the isomorphism (5.2). By Corollary 3.7, the semigroup $\Gamma_{\zeta}$ is isomorphic to the semigroup of Newton polyhedra (with the Minkowski sum) whose elements are $\mathcal{N}(\phi)$ of $0 \neq \phi \in R$ such that $\mathcal{N}(\phi)$ has only one vertex. The Newton polyhedron of $\phi \in R$ is defined in this case as the Newton polyhedron of the image of $\phi$ by the ring extension $R \hookrightarrow \boldsymbol{C}\left\{X_{1}^{1 / n}, \ldots, X_{d}^{1 / n}\right\}$.

The same assertion holds for the semigroup $\Gamma_{\tau}$ with respect to the ring extension $R \hookrightarrow \boldsymbol{C}\left\{Y^{1 / k}, X_{2}^{1 / n}, \ldots, X_{d}^{1 / n}\right\}$. The isomorphism (5.2) induces an isomorphism of semigroups $\Gamma_{\zeta} \cong \Gamma_{\tau}$, since the diagram (5.3) is commutative and the Newton polyhedron of a product is the Minkowski sum of the factors. We deduce from this the following.

Proposition 5.1. The semigroups $\Gamma_{\zeta}$ and $\Gamma_{\tau}$ are isomorphic.

Two hypersurfaces germs $(H, 0)$ and $\left(H^{\prime}, 0\right)$ in $\boldsymbol{C}^{d+1}$ have the same embedded topological type if and only if there is a homeomorphism $U \rightarrow U^{\prime}$, between two open neighbourhoods of the origin, which maps representatives $H \cap U$ to $H^{\prime} \cap U^{\prime}$. The characteristic exponents of a quasi-ordinary branch $\zeta$ determine the embedded topological type of the hypersurface it defines. This is deduced from results of Zariski on saturation of local rings (see $[\mathbf{Z 1}],[\mathbf{L 3}, \S 2]$ and also $[\mathbf{O h}]$ for another proof). Gau, using some topological results of Lipman, proves the equivalence.

Theorem 5.2 (cf. Theorem 1.6 of [Gau]). A pair of analytically irreducible quasiordinary hypersurface germs $(S, 0)$ and $\left(S^{\prime}, 0\right)$ in $\left(\boldsymbol{C}^{d+1}, 0\right)$ have the same topological type if and only if any two normalized quasi-ordinary branches parametrizing $(S, 0)$ and $\left(S^{\prime}, 0\right)$, respectively, have the same characteristic exponents.

We deduce from Proposition 5.1 and Gau's characterization the following result.

Theorem 5.3. If $\zeta$ and $\zeta^{\prime}$ are quasi-ordinary branches parametrizing the same quasiordinary hypersurface, then the semigroups $\Gamma_{\zeta}$ and $\Gamma_{\zeta^{\prime}}$ are isomorphic. The isomorphism class of this semigroup determines, and is determined by, the embedded topological type of the germ $(S, 0)$.

Proof. We can suppose that $\zeta$ and $\zeta^{\prime}$ are given with well-ordered variables. The first assertion follows from Proposition 5.1 and Gau's characterization. Then we can recover from the unique minimal set of generators of the semigroup $\Gamma_{\zeta}$ (by Lemma 3.4) the characteristic exponents of a normalized quasi-ordinary branch parametrizing the same hypersurface germ (see Remark 3.5).

In the case of quasi-ordinary surfaces, the analytical invariance of the semigroup is deduced without using Gau's characterization of the topological type, the analytical invariance of the characteristic monomials being proved by Lipman: he builds a nonembedded resolution of the surface as a composition of monoidal and quadratic transforms that are determined by, and determine, the characteristic monomials of a normalized quasi-ordinary branch parametrizing the surface (see $[\mathbf{L 1}, \mathbf{L 3}]$ ). A direct proof of analytical invariance of the semigroup associated to a quasi-ordinary surface, which implies the invariance properties of the characteristic monomials, has recently been given 
by Popescu-Pampu (see [PP2, PP3]). In the general case, the semigroup appears in an intrinsic manner by analysing, with the generalized Abhyankar's expansions (see Remark 3.10), some algebraic properties of the minimal resolution of the normalization of $S$ at the singular points of the reduced normal crossing divisor defined by the transform of the singular locus of $S$.

Another application of Proposition 5.1 is the following proof of the inversion formulae relating the characteristic exponents of $\zeta$ and $\tau$ (stated in [L1, p. 78] and [L3, p. 170]).

Lemma 5.4. Let $\zeta$ be a quasi-ordinary branch of the form $\zeta=X^{\lambda_{0}} H \in C\left\{\rho^{\vee} \cap M_{g}\right\}$, with $\lambda_{0} \in \rho^{\vee} \cap M$ and $H$ a unit. For any positive integer $k$, the series $\zeta^{k}$ is a quasiordinary branch and its characteristic exponents are $\lambda_{j}^{\prime}=\lambda_{j}+(k-1) \lambda_{0}$ for $j=1, \ldots, g$, where $\left\{\lambda_{j}\right\}_{j=1}^{g}$ are the characteristic exponents of $\zeta$.

Proof. Firstly, we have that $\lambda_{1}^{\prime}<\cdots<\lambda_{g}^{\prime}$. The characteristic lattices $M_{i}$ associated to $\zeta$ coincide with the lattices we can associate to $\left\{\lambda_{j}^{\prime}\right\}_{j=1}^{g}$ by the same formulae. We show that the $\lambda_{j}^{\prime}$ are exponents of $\zeta^{k}$. We expand $\zeta=p_{0}+p_{1}+\cdots+p_{g}$ using Remark 2.3. Each summand

$$
\left(\begin{array}{c}
k \\
s
\end{array}\right)\left(p_{0}+\cdots+p_{j-1}\right)^{k-s}\left(p_{j}+\cdots+p_{g}\right)^{s}
$$

appearing in the binomial expansion of $\left(\left(p_{0}+\cdots+p_{j-1}\right)+\left(p_{j}+\cdots+p_{g}\right)\right)^{k}$ is of the form $X^{(k-s) \lambda_{0}+s \lambda_{j}} \cdot$ unit for $s=0, \ldots, k$, since $\lambda_{0}<\lambda_{1}$ by the hypothesis.

We have $(k-s) \lambda_{0}+s \lambda_{j} \geqslant(k-1) \lambda_{0}+\lambda_{j}=\lambda_{j}^{\prime}$ for $s=1, \ldots, k$, and hence $\zeta^{k}$ is of the form

$$
\zeta^{k}=\left(p_{0}+\cdots+p_{j-1}\right)^{k}+X^{\lambda_{j}^{\prime}} \cdot \text { unit. }
$$

The exponents of the terms appearing in $\left(p_{0}+\cdots+p_{j-1}\right)^{k}$ belong to the group $M_{j-1}$ by Lemma 2.2. Therefore, the monomial $X^{\lambda_{j}^{\prime}}$ appears in $\zeta^{k}$ with non-zero coefficient. It also follows from the formula above that if we have $\lambda_{j-1}^{\prime} \leqslant \lambda$ and $\lambda_{j}^{\prime} \not \leq \lambda$, for $\lambda$ the exponent of a term appearing in $\zeta^{k}$, then this term appears in $\left(p_{0}+\cdots+p_{j-1}\right)^{k}$, and hence $\lambda$ is in the group $M_{j-1}$. The conditions in Lemma 2.2 are satisfied for $\zeta^{k}$ and $\left\{\lambda_{j}^{\prime}\right\}_{j=1}^{g}$.

Proposition 5.5. Suppose that the rational number $\alpha=k / n$ is not an integer. Let $\left\{\lambda_{i}\right\}_{i=1}^{g}$ be the characteristic exponents of $\zeta$. Denote by $\lambda_{i}^{\prime} \in Q^{d}$ the vector with coordinates $\lambda_{i, 1}^{\prime}=\alpha^{-1} \lambda_{i, 1}+\alpha^{-1}-1$ and $\lambda_{i, q}^{\prime}=\lambda_{i, q}$ for $q=2, \ldots, d$ and $i=1, \ldots, g$. Then the characteristic exponents of $\tau$ are

$$
\begin{array}{ll}
\lambda_{1}^{\prime}, \ldots, \lambda_{g}^{\prime} & \text { if } \alpha, \alpha^{-1} \notin \boldsymbol{Z} \\
\lambda_{2}^{\prime}, \ldots, \lambda_{g}^{\prime} & \text { if } \alpha^{-1} \in \boldsymbol{Z} .
\end{array}
$$

Proof. We follow the notations above. Let $m$ be the lowest common denominator of $k$ and $n$. We have $k=u^{\prime} m$ and $n=u m$ for positive integers, $u$, $u^{\prime}$ with $\left(u, u^{\prime}\right)=1$. We consider $U=X_{1}^{1 / u}$ as an indeterminate. As a consequence of Lemma 2.2, the series $\zeta$ defines a quasi-ordinary branch $\zeta_{1}=U^{u^{\prime}} F^{u^{\prime} m}\left(U^{1 / m}, X_{2}^{1 / n}, \ldots, X_{d}^{1 / n}\right)$, whose characteristic exponents $\left\{\lambda_{i}\left(\zeta_{1}\right)\right\}_{i=2}^{g}$ are obtained from $\left\{\lambda_{i}\right\}_{i=2}^{g}$ by multiplying the first coordinate by $u$. 
By Lemma 5.4, the series $\zeta_{2}=U F^{m}\left(U^{1 / m}, X_{2}^{1 / n}, \ldots, X_{d}^{1 / n}\right)$ is a quasi-ordinary branch with characteristic exponents $\left\{\lambda_{i}\left(\zeta_{2}\right)\right\}_{i=2}^{g}$ with coordinates

$$
\lambda_{i, 1}\left(\zeta_{2}\right)=\lambda_{i, 1}\left(\zeta_{1}\right)-u^{\prime}+1=u \lambda_{i, 1}-u^{\prime}+1 \quad \text { and } \quad \lambda_{i, q}\left(\zeta_{2}\right)=\lambda_{i, q}\left(\zeta_{1}\right)=\lambda_{i, q}
$$

for $q=2, \ldots, d$.

We set $V=Y^{1 / u^{\prime}}$ and define

$$
\tau_{1}=V^{u} G^{u m}\left(V^{1 / m}, X_{2}^{1 / n}, \ldots, X_{d}^{1 / n}\right) \quad \text { and } \quad \tau_{2}=V G^{m}\left(V^{1 / m}, X_{2}^{1 / n}, \ldots, X_{d}^{1 / n}\right) .
$$

In the same manner, we deduce that the characteristic exponents, $\left\{\lambda_{i}(\tau)\right\}_{i=2}^{g^{\prime}}$ of $\tau$ that are greater than $\lambda_{1}^{\prime}$, are related to the characteristic exponents $\left\{\lambda_{i}\left(\tau_{2}\right)\right\}_{i=2}^{g^{\prime}}$ of $\tau_{2}$ by $\lambda_{i, 1}\left(\tau_{2}\right)=u^{\prime} \lambda_{i, 1}(\tau)-u+1$ and $\lambda_{i, q}\left(\tau_{2}\right)=\lambda_{i, q}(\tau)$ for $q=2, \ldots, d$.

It is easy to see that $\zeta_{2}=\zeta_{0}^{m}$ and that, by the isomorphism (5.2), $\tau_{2}=\tau_{0}^{m}$ thus restricts to an isomorphism

$$
\boldsymbol{C}\left\{U, X_{2}, \ldots, X_{d}, \zeta_{2}\right\} \rightarrow \boldsymbol{C}\left\{V, X_{2}, \ldots, X_{d}, \tau_{2}\right\} .
$$

Therefore, $\zeta_{2}$ and $\tau_{2}$ parametrize the same hypersurface germ. It follows from the fact that the isomorphism (5.2) preserves Newton polyhedra that the semigroups $\Gamma_{\zeta_{2}}$ and $\Gamma_{\tau_{2}}$ are isomorphic. Since $\zeta_{2}$ and $\tau_{2}$ are both normalized and they have the same semigroup, it follows that they have the same characteristic exponents. This implies that $g=g^{\prime}$ and $\lambda_{i}(\tau)=\lambda_{i}^{\prime}$ for $i=2, \ldots, g$.

If $\alpha^{-1} \in \boldsymbol{Z}$, the characteristic exponents of $\tau$ are $\left\{\lambda_{i}\right\}_{i=2}^{g}$, since $u^{\prime}=1$ and $\tau=\tau_{1}$. Since $\alpha \notin \boldsymbol{Z}$, the first characteristic exponent of $\zeta$ is $\lambda_{1}=(\alpha, 0, \ldots, 0)$ and if $\lambda_{1,1}^{\prime}=\alpha^{-1} \notin \boldsymbol{Z}$, then $\lambda_{1}^{\prime}$ is the first characteristic exponent of $\tau$.

Acknowledgements. I am grateful to B. Teissier and P. Popescu-Pampu for their useful suggestions and discussions. I am grateful to Universidad de la Laguna and Institut de Mathématiques de Jussieu for their hospitality. The author is supported by a Marie Curie Fellowship of the European Community programme 'Improving Human Research Potential and the Socio-economic Knowledge Base' under contract number HPMF-CT2000-00877.

\section{References}

[A1] S. S. Abhyankar, On the ramification of algebraic functions, Am. J. Math. 77 (1955), $575-592$.

[A2] S. S. ABHYANKar, Inversion and invariance of characteristic pairs, Am. J. Math. 89 (1967), 363-372.

[A3] S. S. ABHYANKAR, Irreducibility criterion for germs of analytic functions of two complex variables, Adv. Math. 74 (1989), 190-257.

[A-M1] S. S. AbHyankar and T. Moh, Newton-Puiseux expansion and generalized Tschirnhausen transformation, I, J. Reine Angew. Math. 260 (1973), 47-83.

[A-M2] S. S. Abhyankar and T. Moh, Newton-Puiseux expansion and generalized Tschirnhausen transformation, II, J. Reine Angew. Math. 261 (1973), 29-54.

[Ca] A. Campillo, Algebroid curves in positive characteristic, Lecture Notes in Mathematics, vol. 813 (Springer, 1980). 
[Ew] G. EWALD, Combinatorial convexity and algebraic geometry (Springer, 1996).

[Gau] Y.-N. GAU, Embedded topological classification of quasi-ordinary singularities, Memoirs of the American Mathematical Society, vol. 388 (1988).

[G-T] R. GOLDIN AND B. TEISSIER, Resolving singularities of plane analytic branches with one toric morphism, in Resolution of singularities, Progress in Mathematics, no. 181, pp. 315340 (Birkhäuser, 2000).

[GP1] P. D. GonzÁlez PÉREZ, Singularités quasi-ordinaires toriques et polyèdre de Newton du discriminant, Can. J. Math. 52 (2000), 348-368.

[GP2] P. D. GonzÁLEz PÉREz, Quasi-ordinary singularities via toric geometry, Tesis doctoral, Universidad de La Laguna (2000).

[GP3] P. D. GonzÁlez PÉREz, Toric embedded resolutions of quasi-ordinary singularities, Ann. Inst. Fourier, in press.

[G-P] J. Gwoździewicz And A. Ploski, On the approximate roots of polynomials, Ann. Polonici Math. LX (1995), 199-210.

[J] H. W. E. Jung, Darstellung der Funktionen eines algebraischen Körpers zweier unabhaängigen Veränderlichen $x, y$ in der Umgebung einer stelle $x=a, y=b, J$. Reine Angew. Math. 133 (1908), 289-314.

[Kou] A. G. Kouchnirenko, Polyèdres de Newton et nombres de Milnor, Invent. Math. 32 (1976), 1-31.

[LJ1] M. Lejeune-Jalabert, Contribution a l'étude des singularités de point vue du polygon de Newton thèse, Université de Paris 7 (1973).

[LJ2] M. LEJEune-JALABERT, Sur l'équivalence des singularités des courbes algebroïdes planes (coefficients de Newton), in Introduction à la theorie des singularités I (singularités et monodromie) (ed. Lê Dũng Tráng), pp. 49-154 (Hermann, Paris, 1988).

[L1] J. LIPMAN, Quasi-ordinary singularities of embedded surfaces, Thesis, Harvard University (1965).

[L2] J. LiPMAN, Introduction to resolution of singularities, Proc. Symp. Pure Math. 29 (1975), 187-230.

[L3] J. Lipman, Quasi-ordinary singularities of surfaces in $\boldsymbol{C}^{3}$, Proc. Symp. Pure Math. 40 (1983), 161-172.

[L4] J. LiPMan, Topological invariants of quasi-ordinary singularities, Memoirs of the American Mathematical Society, vol. 388 (1988).

[Lu] I. Luengo, On the structure of embedded algebroid surfaces, Proc. Symp. Pure Math. 40 (1983), 185-193.

[Oh] K. OH, Topological types of quasi-ordinary singularities, Proc. Am. Math. Soc. 117 (1993), 53-59.

[PP2] P. Popescu-Pampu, Arbres de contact des singularités quasi-ordinaires et graphes d'adjacence pour les 3-variétés réelles, Thèse de doctorat, Université de Paris 7 (2001).

[PP3] P. POPESCU-PAMPU, On the invariance of the semigroup of a quasi-ordinary surface singularity, C. R. Acad. Sci. Paris Sér. I 334 (2002), 1101-1106.

[PP1] P. Popescu-PAmpu, Approximate roots, in Valuation theory and its applications, vol. 2, Proc. Int. Conf. Workshop on Valuation Theory, Saskatoon, Canada 1999, in press.

[Re] J. E. REEVE, A summary of results on the topological classification of plane algebroid singularities, Rend. Sem. Mat. Univ. Politec. Torino 14 (1954), 159-187.

[T] B. TeIssien, The monomial curve and its deformations, appendix in [Z2].

[W] R. J. WALkER, Reduction of the singularities of an algebraic surface, Ann. Mat. 36 (1935), 336-365.

[Z2] O. ZARISKI, Le problème des modules pour les branches planes (Hermann, Paris, 1986).

[Z1] O. ZARISKI, Studies in equisingularity, III, Saturation of local rings and equisingularity, Collected Papers, vol. 4, pp. 96-158, Equisingularity on algebraic varieties (MIT Press, Cambridge, MA, 1979). 